\title{
Ovarian insufficiency and early pregnancy loss induced by activation of the innate immune system
}

\author{
Adrian Erlebacher, ${ }^{1,2}$ Dorothy Zhang, ${ }^{1}$ Albert F. Parlow, ${ }^{3}$ and Laurie H. Glimcher ${ }^{1,2}$ \\ 1Department of Immunology and Infectious Diseases, Harvard School of Public Health, Boston, Massachusetts, USA. \\ 2Department of Medicine, Harvard Medical School, Boston, Massachusetts, USA. ${ }^{3}$ National Hormone and Peptide Program, \\ Harbor-University of California Los Angeles Medical Center, Torrance, California, USA.
}

\begin{abstract}
We describe a murine model of early pregnancy failure induced by systemic activation of the CD40 immune costimulatory pathway. Although fetal loss involved an NK cell intermediate, it was not due to lymphocytemediated destruction of the fetus and placenta. Rather, pregnancy failure resulted from impaired progesterone synthesis by the corpus luteum of the ovary, an endocrine defect in turn associated with ovarian resistance to the gonadotropic effects of prolactin. Pregnancy failure also required the proinflammatory cytokine TNF- $\alpha$ and correlated with the luteal induction of the prolactin receptor signaling inhibitors suppressor of cytokine signaling 1 (Socs1) and Socs3. Such links between immune activation and reproductive endocrine dysfunction may be relevant to pregnancy loss and other clinical disorders of reproduction.
\end{abstract}

\section{Introduction}

The mechanisms underlying immune-mediated pregnancy failure remain largely unknown (1). In women with recurrent pregnancy loss, increased numbers and cellular activation of peripheral blood $\mathrm{CD}_{56}{ }^{+} \mathrm{NK}$ cells have suggested an important role for the innate immune system (2-6). Since NK cells produce the signature Th1 cytokine IFN- $\gamma$, NK cell activation during pregnancy might explain the apparent Th1 bias also associated with recurrent pregnancy loss $(7,8)$. These associations, however, have not led to a clear understanding of the ultimate cause of fetal demise. Data from rodent studies have suggested that the major mechanism of immune-mediated pregnancy failure is immune activation at the maternal-fetal interface. Activation of decidual immune cells with attendant inflammatory cytokine production or activation of complement via the binding of anti-phospholipid antibodies is thought to directly damage the fetus and placenta or lead to derangements in decidual or placental hemostasis (1, 9-11).

The possibility that immune activation might cause pregnancy failure by inhibiting the reproductive endocrine system has been largely unexplored, despite indications that immune processes regulate reproductive endocrine function in nonpregnant mammals. Inflammatory cytokines are thought to inhibit gonadotropin production at the level of the hypothalamus and pituitary in cases of chronic or acute illness (12), and to inhibit progesterone synthesis by the corpus luteum of the ovary and promote luteal regression as part of the normal ovulatory cycle (13). During early gestation in rodents, ovarian progesterone production is driven primarily by pituitary-derived prolactin binding to its Janus kinase 2/signal

Nonstandard abbreviations used: C57BL/6 (B6); embryonic day (E); human chorionic gonadotropin (hCG); $3 \beta$-hydroxysteroid dehydrogenase (3 $\beta$-HSD); $20 \alpha$-hydroxysteroid dehydrogenase (20 $\alpha$-HSD); Janus kinase (JAK); luteinizing hormone (LH); LH receptor (LHR); P450 side chain cleavage enzyme (P450scc); signal transducer and activator of transcription (STAT); steroidogenic acute regulatory protein (StAR); suppressor of cytokine signaling (Socs).

Conflict of interest: The authors have declared that no conflict of interest exists.

Citation for this article: J. Clin. Invest. 114:39-48 (2004).

doi:10.1172/JCI200420645. transducer and activator of transcription 5-coupled (JAK2/STAT5coupled) receptor expressed by corpus luteal cells (14). The binding of progesterone to its nuclear receptor, in turn, is thought to maintain decidual viability and inhibit myometrial contractility $(15,16)$. In addition, there is strong evidence that luteinizing hormone (LH) produced by the pituitary is also critical for maintaining luteal function in rats early in pregnancy (17), and this is likely also to be the case in mice (18). Thus, the reproductive endocrine system provides multiple potential points for inhibition by the immune system.

We describe a new link between the immune system and the reproductive endocrine system that is regulated by the TNF receptor superfamily member CD40, an immune costimulatory molecule whose expression on dendritic cells is critical for the initiation of cell-mediated immune responses $(19,20)$. Forced ligation of CD40 with agonistic anti-CD40 antibodies caused a systemic inflammatory response that efficiently induced embryo resorption in mice early in gestation. However, pregnancy failure occurred not through immune cell activation at the maternal-fetal interface, but rather through decreased progesterone synthesis by the corpus luteum. Luteal insufficiency was induced largely by activated NK cells, required the actions of the proinflammatory cytokine TNF- $\alpha$, and was associated with impaired prolactin receptor signaling. Furthermore, luteal insufficiency correlated with the induction of suppressor of cytokine signaling 1 (Socs1) and Socs3, two members of the Socs family of proteins that provide critical feedback inhibition of JAK/STAT signaling at the immediate postreceptor level (21-23). The gestational effects of CD40 ligation delineate an in vivo pathway whereby activation of the innate immune system with associated inflammatory cytokine production leads to pregnancy failure through inhibition of the reproductive endocrine system.

\section{Results}

Systemic CD40 ligation in mice causes early pregnancy failure preceded by decreased serum progesterone concentrations. To determine the gestational effects of systemic CD40 ligation, we injected the agonistic anti-CD40 mAb FGK45 (24) at various times during pregnancy. Four daily injections of FGK45 from embryonic day 4 (E4) to E7 
Table 1

Systemic CD40 ligation causes murine pregnancy failure in the postimplantation period

\begin{tabular}{|c|c|c|c|c|c|c|c|c|}
\hline Treatment period & Female & Male & Treatment & Sacrifice & $n$ & Number pregnant & Pregnancy rate $(\%)$ & Litter size $( \pm$ SD) \\
\hline \multirow[t]{6}{*}{ E4-7 } & $\mathrm{BALB} / \mathrm{C}$ & $\mathrm{BALB} / \mathrm{c}$ or $\mathrm{B} 6^{\mathrm{A}}$ & Rat $\lg G$ & E17 & 25 & 14 & 56 & $7.2 \pm 1.7$ \\
\hline & & & Anti-CD40 & E17 & 25 & 1 & $4^{C}$ & 9 \\
\hline & B6 & B6 & Rat IgG & E14-17 & 12 & 5 & 42 & $8.6 \pm 0.9$ \\
\hline & & & Anti-CD40 & E14-17 & 11 & 0 & $0^{D}$ & NA \\
\hline & B6CBAF1 & B6 & Rat IgG & E8 & 15 & 15 & 100 & $9.3 \pm 1.3$ \\
\hline & & & Anti-CD40 & E8 & 22 & 1 & $5^{C}$ & 8 \\
\hline \multirow[t]{4}{*}{ E8-11 } & $\mathrm{BALB} / \mathrm{c}$ & $\mathrm{BALB} / \mathrm{C}$ or $\mathrm{B} 6^{\mathrm{B}}$ & Rat $\lg G$ & E17 & 11 & 4 & 36 & $6.5 \pm 1.7$ \\
\hline & & & Anti-CD40 & E17 & 12 & 6 & 50 & $7.0 \pm 1.8$ \\
\hline & B6CBAF1 & B6 & Rat IgG & E12 & 7 & 6 & 86 & $6.9 \pm 2.3$ \\
\hline & & & Anti-CD40 & E12 & 6 & 6 & 100 & $7.9 \pm 1.1$ \\
\hline
\end{tabular}

AMales were divided between 12 BALB/C and 13 C57BL/6 (B6) mice for both treatment groups. BMales were divided between five to six BALB/C and five to six $\mathrm{B} 6$ mice for both treatment groups. ${ }^{\mathrm{C} P}<0.0001$. $\mathrm{D} P<0.02$.

dramatically reduced the percentage of pregnant mice per total mated mice to nearly $0 \%$ in females of all strains tested, with control pregnancy rates after treatment with polyclonal rat IgG varying between $40 \%$ and $100 \%$ depending on the background strain (Table 1). The effect of FGK45 injection occurred in both syngeneic and allogeneic mating combinations and was restricted to the immediate postimplantation period, since similar daily injections on E8-11 did not reduce pregnancy rates or litter sizes. FGK45 injection also did not inhibit pregnancy in $\mathrm{Cd}_{40^{-/}-}$females (25) mated to wild-type males, ruling out the possibility that its abortifacient effect was nonspecific or due to embryonic or placental ligation of CD40 (data not shown). Rather, pregnancy failure required maternal CD40 expression.

Although CD40 is largely considered an immune stimulatory molecule, the timing of events leading to embryo loss suggested that the ultimate cause of pregnancy failure following CD40 ligation was endocrinological rather than immunological. Using B6CBAF1 females to take advantage of their high natural pregnancy rate (Table 1), we found that a single FGK45 injection on E4 dramatically reduced serum progesterone levels within 24 hours (Figure 1A), yet implantation sites from these mice showed no evidence of a pathological leukocyte infiltration that might be expected with a tissue-rejection response. Rather, compared with controls (Figure 1, B and D), implantation sites from FGK45-treated mice were only mildly reduced in size at E5 (Figure 1C) and contained implanted embryos with the typical clearing of $\mathrm{CD} 45^{+}$leukocytes (red) from the early primary maternal decidua (Figure 1E). By E8, however, after daily FGK45 injections on E4-7, all implantation sites had been completely resorbed (see below), and serum progesterone levels remained low (Figure 1A). Since progesterone is the sole ovarian hormone required for the maintenance of postimplantation pregnancy (26), its reduced concentration in serum prior to overt embryo resorption suggested that CD40 ligationinduced abortion involved an inhibition of the
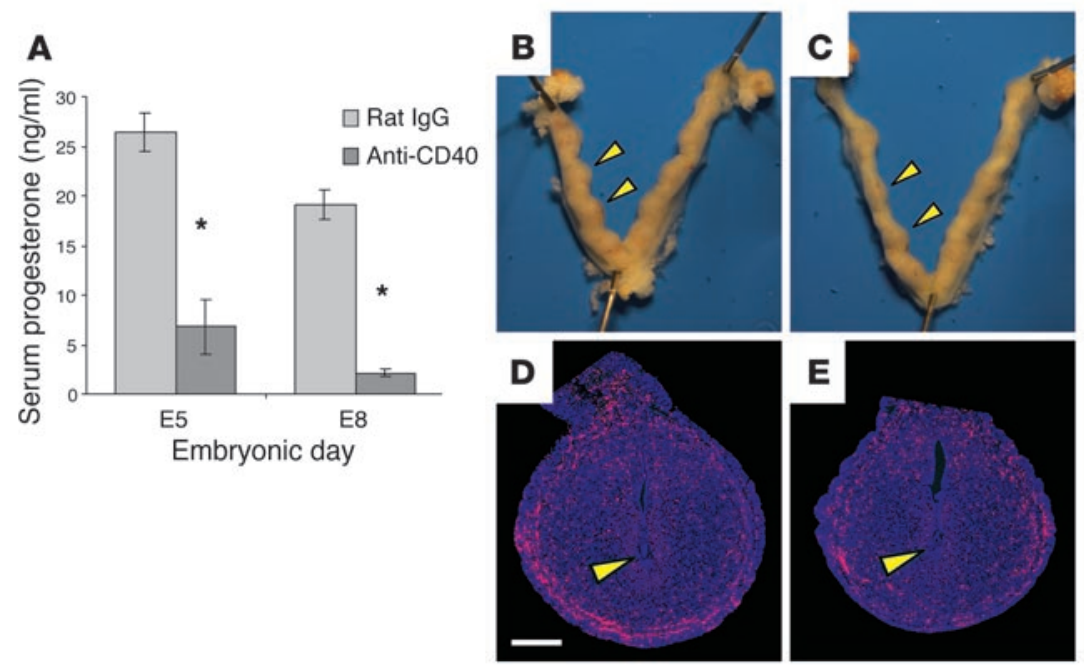

\section{Figure 1}

Decreased serum progesterone concentrations following systemic CD40 ligation precedes overt embryo resorption. Mice either were treated with control rat IgG or agonistic anti-CD40 antibodies (FGK45) once on E4 and sacrificed 24 hours later on E5, or were treated daily on E4-7 and sacrificed on E8. (A) Serum progesterone concentrations. Data represent mean \pm SEM of six mice per group. Progesterone concentrations in FGK45treated mice were significantly reduced compared with those in control mice. ${ }^{*} P<0.001$. (B-E) Implantation sites on E5 from mice treated with rat IgG (B and D) or FGK45 (C and E). (B and C) Whole-mount preparations of uteri, with arrowheads indicating two implantation sites. ( $\mathbf{D}$ and $\mathbf{E}$ ) Paraffin sections of implantation sites stained with anti-CD45 antibodies to visualize all leukocytes (red), and counterstained with DAPI to visualize all cell nuclei (blue). The mesometrial pole of each implantation site is oriented toward the top of the panel. A recently implanted embryo can be seen in the center of each section (arrowhead). Immunostaining is representative of two to three mice per group, encompassing a total of 15-20 implantation sites in each group. Scale bar: $0.5 \mathrm{~mm}$. 

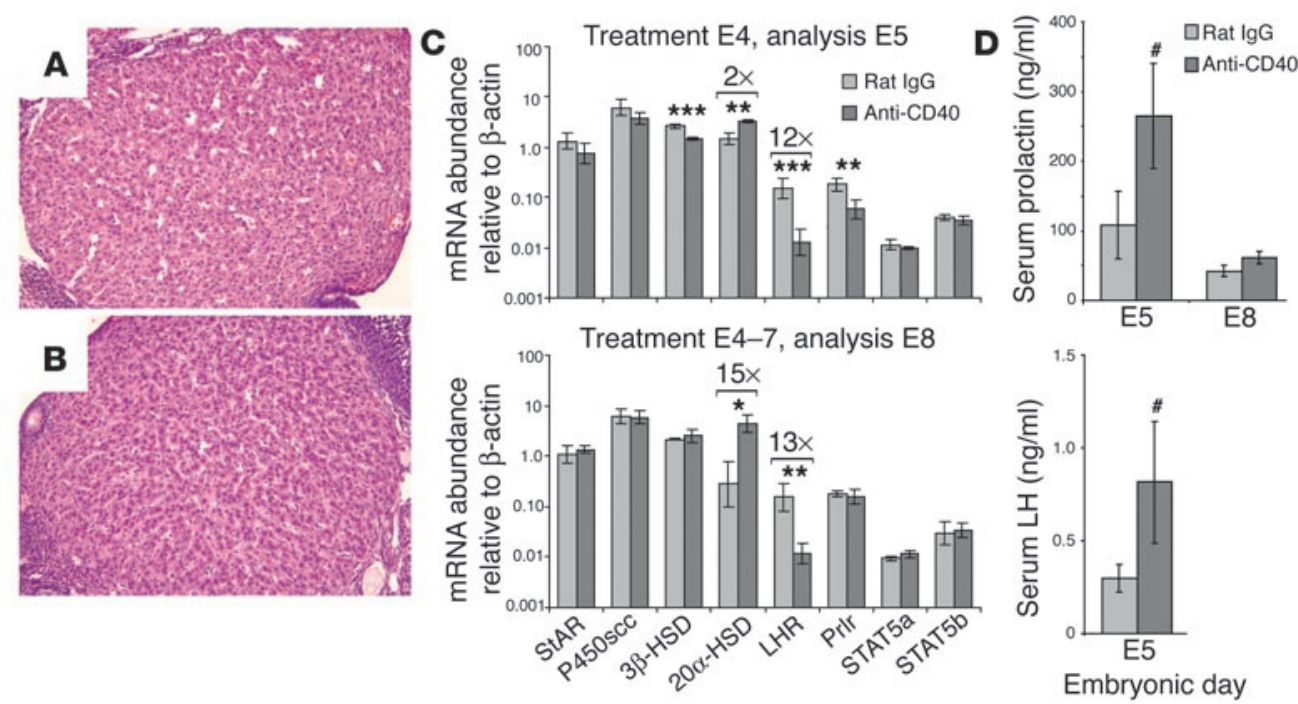

\section{Figure 2}

Postreceptor prolactin signaling defects and luteal insufficiency following systemic CD40 ligation. (A and B) Histological appearance of corpora lutea from mice on E8 following daily E4-7 injections of rat IgG (A) or anti-CD40 antibodies (FGK45) (B). Paraffin sections stained with H\&E are shown. (C) Whole-ovary mRNA expression levels determined by real-time RT-PCR. Ovaries were collected from mice that either had been treated with control rat IgG or anti-CD40 antibodies (FGK45) once on E4 and sacrificed 24 hours later on E5 ( $n=4$ mice per group; upper panel), or had been treated daily on E4-7 and sacrificed on E8 ( $n=3$ mice per group; lower panel). Data represent mean \pm SD. On both E5 and E8, $20 \alpha-H S D$ and LHR mRNA levels were increased and decreased, respectively (fold changes are indicated by brackets), whereas $3 \beta-H S D$ and prolactin receptor (Prlr) mRNA levels were decreased on E5 only. ${ }^{*} P<0.02$; ${ }^{* *} P<0.005$; ${ }^{* * *} P<0.001$. (D) Serum prolactin and LH concentrations. Prolactin and LH concentrations in FGK45-treated mice on E5 were significantly elevated compared with those in control mice $(\# P<0.05$; data represent mean \pm SEM of 12 mice per group), but there was no change in prolactin concentrations on E8 ( $n=6$ mice per group). The limit of detection for LH was $0.2 \mathrm{ng} / \mathrm{ml}$.

(Figure 2A), there was little microscopic evidence that CD40 ligation caused the histological regression of corpora lutea (i.e., structural luteolysis), since these structures were still clearly present on E8 following daily FGK45 injections on E4-7 and only showed a mild and variable increase in luteal cellularity (Figure 2B). Furthermore, quantitative real-time RT-PCR analysis of ovarian RNA from FGK45-treated mice (Figure $2 \mathrm{C}$ ) revealed no significant change on either E5 or E8 in the levels of mRNA encoding the steroidogenic enzymes steroidogenic acute regulatory protein (StAR) and P450 side chain cleavage enzyme (P450scc), and only a transient twofold decrease on E5 alone in the mRNA level of $3 \beta$-hydroxysteroid

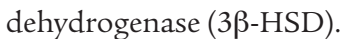

On the other hand, CD40 ligation induced dramatic changes in the mRNA expression levels of the luteinizing hormone receptor (LHR) and $20 \alpha$-HSD, the two major transcriptional targets of prolactin receptor signaling in corpus luteal cells $(14,27)$ (Figure 2C). Normally, prolactin receptor signaling via JAK2/STAT5 induces the transcription of LHR and represses the transcription of $20 \alpha-$ HSD (14). However, a single FGK45 injection on E4 led to a 12-fold decrease in the abundance of LHR mRNA and a twofold increase in $20 \alpha$-HSD mRNA within 24 hours, which progressed after daily FGK45 injections on E4-7 to a 13-fold decrease and a 15-fold increase in mRNA transcript levels, respectively, on E8. Thus, CD40 ligation led to a functional luteal insufficiency associated with defective prolactin receptor target-gene expression. Since $20 \alpha$-HSD catalyzes the intraovarian catabolism of newly synthesized progesterone (14), its derepression in FGK45-treated mice likely contributed to the decrease in serum progesterone concentrations.

Further molecular and hormonal analyses suggested that the defect in prolactin receptor target-gene expression was due to inhibition at the postreceptor level. Thus, serum levels of prolactin were actually modestly elevated from those of controls 24 hours after a single FGK45 injection on E4, consistent with a loss of feedback inhibition by progesterone (13), and unimpaired pituitary function was also suggested by the modest elevation in serum LH levels on E5 (Figure 2D). On E8, after daily E4-7 FGK45 injection, serum prolactin levels were similar to those of controls (Figure 2D), with the overall increase in prolactin levels at this later time point consistent with the known pattern of pituitary prolactin secretion during early mouse gestation (28). Furthermore, we observed only a transient threefold decrease in prolactin receptor mRNA expression on E5 alone (due to proportionate decreases in mRNAs for the long and short isoforms; data not shown) and normal expression levels on E8, with unaltered mRNA expression of STAT5a and STAT5b on both E5 and E8 (Figure 2C). Collectively, these data implied that the deregulation of prolactin target genes was due to the presence of a dominant inhibitor of STAT5 signaling, a possibility we explore further below.

To directly evaluate whether luteal insufficiency was the ultimate cause of pregnancy failure, we treated pregnant mice with both FGK45 and progesterone. Strikingly, daily progesterone administration on E4-7, which led to serum concentrations of about $35 \mathrm{ng} / \mathrm{ml} 24$ hours after the E7 injection, almost completely prevented abortions caused by daily E4-7 FGK45 injection (Table 2). Implantation sites at E8 from dually treated mice appeared completely normal both grossly (Figure 3G) and histologically (Figure $3 \mathrm{H}$ ) and showed no evidence of an altered distribution of $\mathrm{CD} 45^{+}$leukocytes (red-stained cells) compared with those from control mice treated with either rat IgG plus progesterone (Figure 3, E and F) or rat IgG plus sesame seed oil vehicle (Figure 3, A and 


\section{Table 2}

Rescue of CD40 ligation-induced pregnancy failure by exogenous progesterone or prolactin

\begin{tabular}{|c|c|c|c|c|c|}
\hline Treatment & Cotreatment & $n$ & $\begin{array}{l}\text { Number } \\
\text { pregnant }\end{array}$ & $\begin{array}{c}\text { Pregnancy } \\
\text { rate (\%) }\end{array}$ & $\begin{array}{l}\text { Litter size } \\
\text { ( } \pm \text { SD) }\end{array}$ \\
\hline \multirow[t]{2}{*}{ Rat IgG } & Vehicle & 10 & 10 & 100 & $9.2 \pm 0.9$ \\
\hline & Progesterone & 13 & 12 & 92 & $9.5 \pm 1.5$ \\
\hline \multirow[t]{2}{*}{ Anti-CD40 } & Vehicle & 10 & 0 & 0 & NA \\
\hline & Progesterone & 13 & 12 & $92^{A}$ & $9.2 \pm 1.5$ \\
\hline \multirow[t]{2}{*}{ Anti-CD40 } & Vehicle & 5 & 0 & 0 & $8.6 \pm 0.9$ \\
\hline & Ovine prolactin & 8 & 6 & $75^{B}$ & $9.0 \pm 2.0$ \\
\hline
\end{tabular}

Mice were sacrificed on E8 following daily E4-7 treatment with rat IgG or anti-CD40 antibodies (FGK45) plus cotreatment either with progesterone or sesame seed oil vehicle, or with prolactin or alkaline saline vehicle. ${ }^{A} P<0.0001$. ${ }^{B} P<0.01$.

B). In contrast, E8 uteri of mice treated with anti-CD40 antibodies plus vehicle showed no evidence of implantation (Figure 3C), occasionally contained debris in the uterine lumen (Figure 3D, arrowhead), and by this end stage were infiltrated with $\mathrm{CD} 45^{+}$leukocytes scattered throughout the uterine stroma. Continued daily progesterone injection, up to E17, also maintained pregnancy in mice treated with FGK45 on E4-7 (six of six mice).

In addition to progesterone, twice-daily coadministration of 100 $\mu \mathrm{g}$ ovine prolactin prevented CD40 ligation-induced abortion (Table 2), and implantation sites from these mice also showed no histological abnormalities (data not shown). Prolactin injections raised serum progesterone concentrations above those seen in mice treated with FGK45 alone; however, these levels still remained subphysiological $(10.9 \pm 1.5 \mathrm{ng} / \mathrm{ml}$ [mean $\pm \mathrm{SEM}]$ on E8). Thus, although the corpora lutea of FGK45-treated mice were resistant to physiological or modestly elevated serum prolactin levels, this could be overcome by exogenous prolactin administration at high doses.

Induction of luteal insufficiency requires a lymphoid intermediate and TNF- $\alpha$ and is associated with increased ovarian Socs 1 and Socs 3 expression. To understand how systemic CD40 ligation caused luteal insufficiency, we first used flow cytometry to determine that there was no significant CD40 staining of enzymatically dispersed E4 ovarian cell suspensions aside from a subset of $\mathrm{CD} 45^{+}$leukocytes (data not shown). This ruled out the possibility that FGK45 treatment induced abortion via direct ligation of CD40 on corpus luteal cells themselves. Furthermore, CD40 ligation induced abortion in

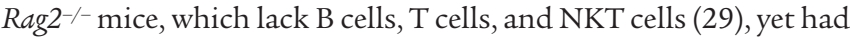
no effect on pregnancy in completely alymphoid $\mathrm{Rag}^{2^{-/-} \gamma_{c}^{-/-}}$mice, which additionally lack NK cells (30) (Table 3). Thus, a lymphocyte population was required for the abortifacient effects of CD40 ligation, and NK cells were sufficient to perform this function.

As expected, systemic CD40 ligation led to broad activation of lymphocytes and dendritic cells. Importantly, this activation was not affected by concurrent progesterone administration, which suggested that it lay entirely upstream of luteal insufficiency. Thus, we found the same degree of splenomegaly (Figure 4A), increased lymphocyte cell size (Figure 4B), modulated expression of the lymphoid activation markers CD69 (Figure 4B), CD25, CD44, CD45RB, and CD62L (data not shown), and upregulation of the costimulatory molecules CD80, CD86, MHC class II (Figure 4D), CD40, and CD54 (data not shown) on splenic CD11 $\mathrm{c}^{+}$ dendritic cells. The splenic CD $11 b^{+} \mathrm{CD} 11 \mathrm{c}^{-}$macrophage popula-

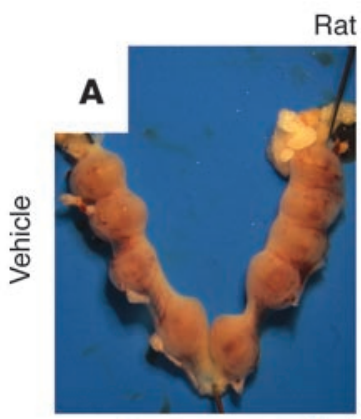

Rat $\lg G$
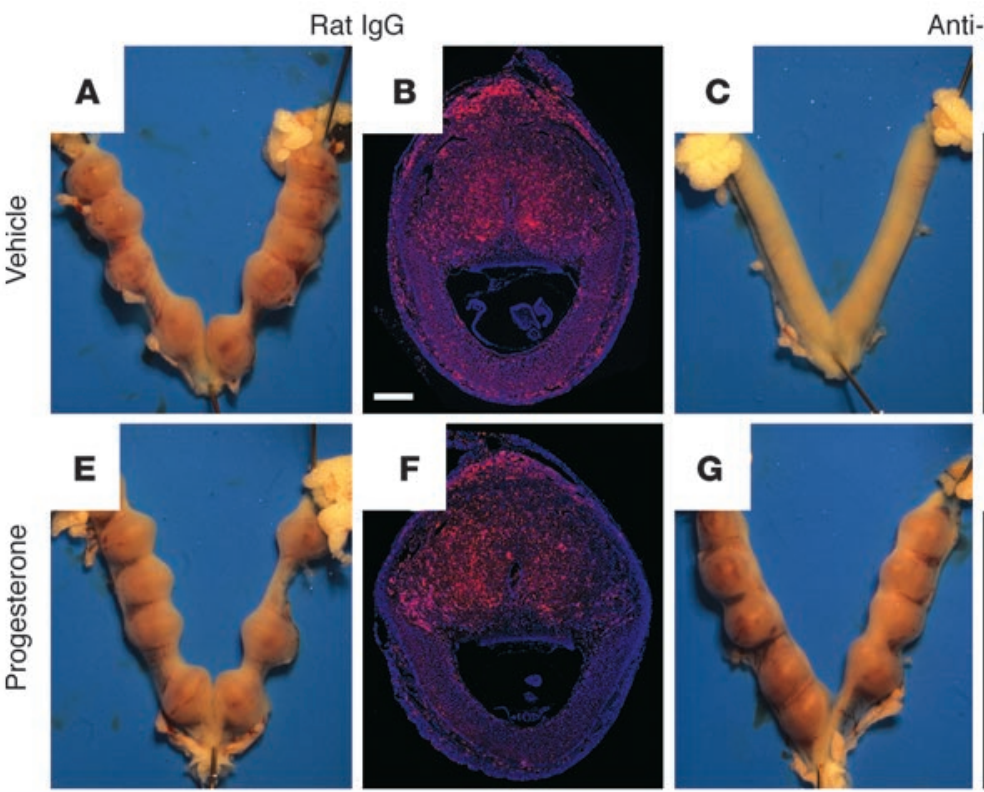

Anti-CD40
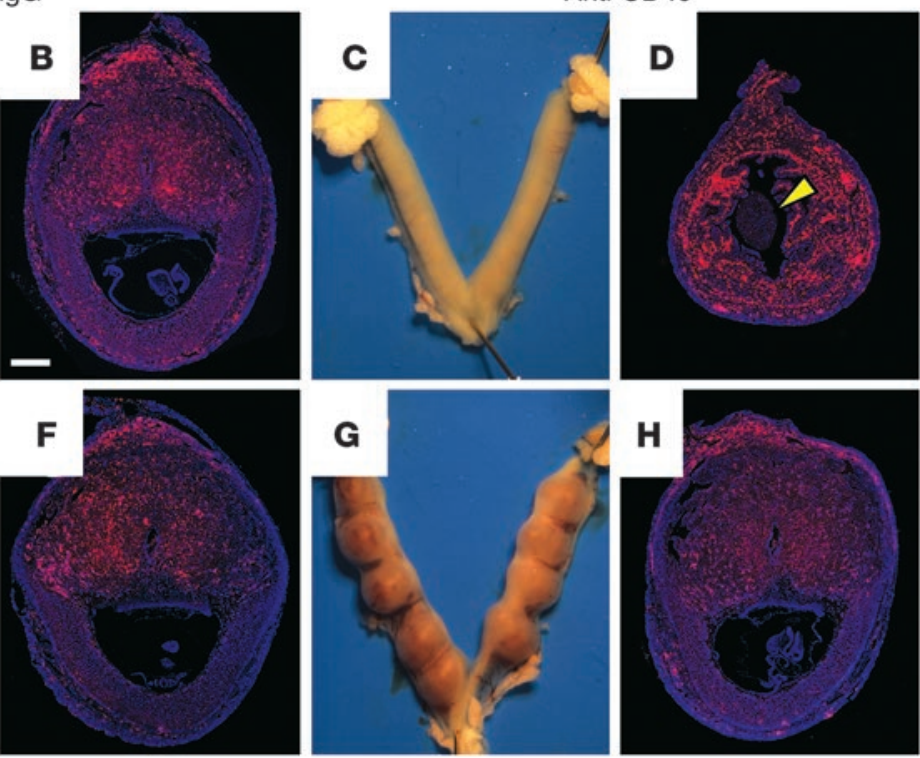

Figure 3

Rescue of CD40 ligation-induced pregnancy failure by exogenous progesterone. (A-H) Whole-mount uteri (A, C, E, and G) and implantation-site histology (B, D, F, and $\mathbf{H}$ ) from mice on E8 following daily treatments on E4-7. Paraffin sections of implantation sites were stained with anti-CD45 antibodies to visualize all leukocytes (red), and counterstained with DAPI (blue). Only mice treated with FGK45 and sesame seed oil vehicle (C and D) showed abortion, leukocytic infiltration into the uterine stroma, and occasional cell debris in the uterine lumen (D, arrowhead). The CD45 staining intensity in these sections tended to be greater than that at viable implantation sites, because they do not contain the high numbers of CD45 dim uterine NK cells that are present in the decidua. Immunostaining is representative of two mice per group, encompassing a total of about 12 implantation sites in each group. Scale bar: $0.5 \mathrm{~mm}$. 
Table 3

Genetic analysis of lymphoid requirements for pregnancy failure following CD40 ligation

\begin{tabular}{lccccc} 
Female & Treatment & $\boldsymbol{n}$ & $\begin{array}{c}\text { Number } \\
\text { pregnant }\end{array}$ & $\begin{array}{c}\text { Pregnancy } \\
\text { rate (\%) }\end{array}$ & $\begin{array}{c}\text { Litter size } \\
( \pm \text { SD) }\end{array}$ \\
Rag2-- $^{2-}$ & Rat IgG & 9 & 5 & 56 & $8.4 \pm 4.2$ \\
Rag2 $^{-/} \gamma_{C}{ }^{-/}$ & Anti-CD40 & 9 & 0 & 0 & NA \\
& Rat IgG & 6 & 4 & 67 & $8.0 \pm 2.4$ \\
& Anti-CD40 & 5 & 4 & 80 & $8.0 \pm 1.6$ \\
\hline
\end{tabular}

Mice were sacrificed on E8 following daily E4-7 treatment with rat IgG or anti-CD40 antibodies (FGK45). Data for control C57BL/6 matings are shown in Table 1. ${ }^{A} P<0.01$; an equivalent effect was seen with Rag2 $^{-/-}$females on a BALB/c background.

tion expressed low levels of CD40 and showed only modest changes in surface marker expression after daily E4-7 FGK45 treatment (data not shown).

Since FGK45 injection did not cause a significant leukocytic infiltration into the ovaries (data not shown), luteal insufficiency following CD40 ligation was likely due to inflammatory cytokines produced by the immune system potentially acting at the systemic level. In contrast to unaltered serum levels of IL-1 $\beta$, IL-2, IL-4, IL-10, IL-12, or IFN- $\gamma$, a single FGK45 injection on E4 induced a transient increase after 24 hours in the serum concentrations of TNF- $\alpha$ and its downstream target IL-6 (Figure 5A) (31). Indeed, TNF- $\alpha$ was critical for pregnancy failure, as its neutralization with a specific $\mathrm{mAb}$ prevented FGK45-induced abortion (Table 4). Furthermore, immunohistochemical and biochemical studies suggested that the cytokine was acting directly on corpus luteal cells. Thus, the p65 subunit of NF- $\mathrm{kB}$, a transcriptional mediator of TNF receptor signaling present in the cytoplasm of unstimulated cells (Figure 5B), had assumed a nuclear distribution pattern in corpus luteal cells after FGK45 injection on E4 (Figure 5C). FGK45 injection also led to 11-fold and twofold respective increases in the ovarian abundances of transcripts for IL- 6 and the additional

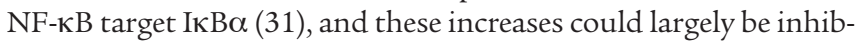
ited by administration of TNF- $\alpha$-neutralizing antibodies (Figure 6). Collectively, these data show that TNF- $\alpha$ was required for FGK45-induced pregnancy failure and suggest that corpus luteal

\section{Figure 4}

Systemic immune activation by CD40 ligation is unaffected by progesterone administration. Mice were sacrificed on E8 after daily treatment on E4-7 with rat IgG or FGK45 plus either concurrent progesterone injection (which produced no abortion with either rat IgG or FGK45) or concurrent sesame seed oil vehicle injection (which produced abortion with FGK45 only). (A) Elevated splenocyte numbers following FGK45 treatment in both groups. ${ }^{\star} P<0.005$. Data represent mean \pm SD for three to four mice per group. (B-D) Three-color flow cytometric analysis of splenocytes to determine cell size and surface activation marker expression. In all panels, shaded histograms show data from rat IgGtreated mice, while open histograms show data from FGK45-treated mice. Data represent three mice per group. (B and $\mathbf{C})$ Lymphocyte activation. Increased forward scatter (FSC), indicating increased cell size (B), and increased CD69 expression, indicating cell activation (C), were seen in B cells, NK cells, and a fraction of T cells following FGK45 treatment in both groups. (D) Dendritic cell activation. Increased CD80, CD86, and MHC class II expression, indicating cell activation, was seen on splenic CD11c cells following FGK45 treatment in both groups. cells were functionally exposed to this cytokine during the course of FGK45-induced abortion. The ovary was also likely exposed to IL-6 as a secondary consequence of its systemic or local induction.

Both TNF- $\alpha$ and IL- 6 are known to transcriptionally induce multiple members of the Socs family of JAK/STAT signaling inhibitors $(22,23)$. While only assessed to date outside the context of pregnancy, Socs induction is thought to be the major mechanism of cross-talk inhibition between JAK/STAT signaling cascades of the immune and endocrine systems (21-23). By analogy, the ovarian exposures to TNF- $\alpha$ and IL- 6 directly suggested Socs proteins as the dominant inhibitors of STAT5 signaling that we inferred to exist from our expression studies of ovarian steroidogenic enzymes and prolactin receptor signaling components (see above). Indeed, 12 hours after a single FGK45 injection on E4, ovarian Socs1 and Socs 3 mRNA levels were increased 11-fold and twofold, respectively (Figure 6), while Socs2 mRNA levels remained unchanged (data not shown). Compared with mice treated with FGK45 plus control hamster IgG mAb's, mice treated with FGK45 plus TNF- $\alpha$-neutralizing antibodies showed partially inhibited Socs1 induction and largely inhibited Socs3 induction (Figure 6). Furthermore, Socs 1 and Socs 3 inducibility on E4 correlated with the E4-7 temporal window of sensitivity toward FGK45-induced abortion,
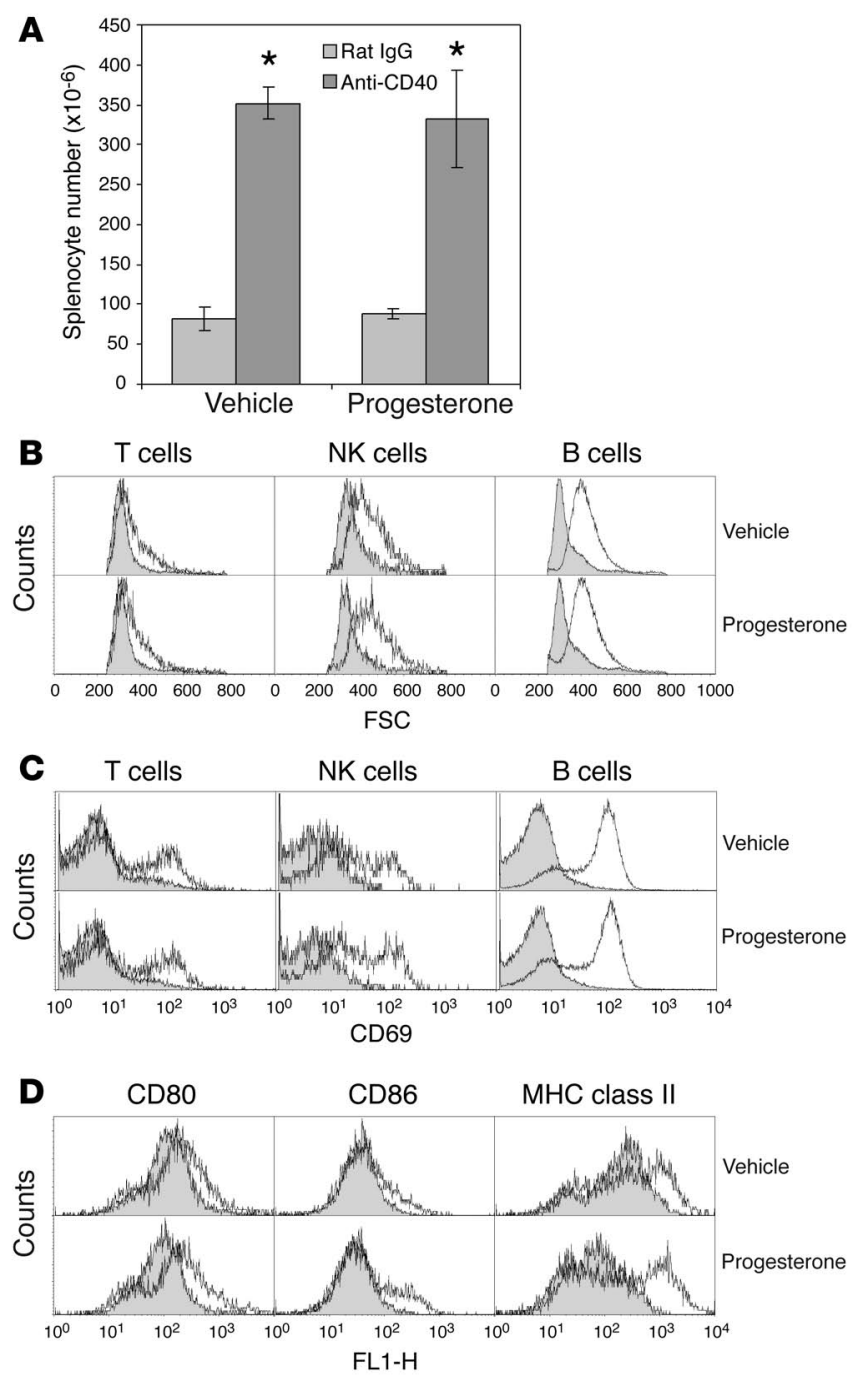


\section{Table 4}

TNF- $\alpha$ blockade prevents FGK45-induced pregnancy failure

\begin{tabular}{|c|c|c|c|c|c|}
\hline Treatment & Cotreatment & $n$ & $\begin{array}{l}\text { Number } \\
\text { pregnant }\end{array}$ & $\begin{array}{c}\text { Pregnancy } \\
\text { rate (\%) }\end{array}$ & $\begin{array}{l}\text { Litter size } \\
\text { ( } \pm \text { SD) }\end{array}$ \\
\hline $\mathrm{t} \lg$ & Hamster Ig & & 6 & 100 & $8.2 \pm 1.2$ \\
\hline 40 & Hamster IgG & . & 0 & $\mathrm{OA}^{\mathrm{A}}$ & \\
\hline nti-CD40 & Anti-TNF- $\alpha$ & 7 & 6 & $86^{B}$ & $8.5 \pm 1.2$ \\
\hline
\end{tabular}

Mice were sacrificed on E8-10 after E4-7 injections of rat IgG and control hamster IgG mAb's, FGK45 and control hamster IgG mAb's, or FGK45 and TNF- $\alpha-$ neutralizing hamster IgG mAb's. One mouse receiving FGK45 and TNF- $\alpha$-neutralizing antibodies was allowed to carry its litter to term. ${ }^{A} P<0.001$ vs. rat IgG plus hamster IgG. ${ }^{B} P<0.005$ vs. anti-CD40 plus hamster lgG.

since FGK45 injection on E8 did not significantly increase Socs 1 or Socs3 mRNA expression (Figure 6). Base-line ovarian Socs1 and Socs 3 expression was higher at this later point in gestation. The failure to induce Socs 1 and Socs 3 was likely due to altered ovarian responsiveness on $\mathrm{E} 8$, since the pattern of immune cell surface marker modulation following FGK45 injection was identical in the E8-11 and E4-7 periods (see above, and data not shown), and similar levels of IL- 6 and IKB $\alpha$ mRNA induction were seen 12 hours after FGK45 injection on E8 versus E4 (Figure 6). Lastly, progesterone treatment did not affect the FGK45-induced ovarian upregulation of IL-6, IкB $\alpha$, Socs 1 , or Socs 3 expression (Figure 6), consistent with immune activation acting entirely upstream of luteal insufficiency. Together, these data suggest that the acute inductions of Socs 1 and Socs 3 are important mechanisms of deregulated luteal prolactin target-gene expression and decreased ovarian progesterone synthesis following systemic CD40 ligation.

\section{Discussion}

We describe a model of early pregnancy loss in mice that links the immune system with the reproductive endocrine system. In this model, forced ligation of CD40 with the agonistic anti-CD40 mAb FGK45 stimulates dendritic cells and initiates a systemic inflammatory response. This inflammatory response in turn induces an ovarian resistance toward the gonadotropic effects of pituitary-derived prolactin and thus ultimately causes pregnancy failure by inhibiting ovarian progesterone production. The link between immune activation and endocrine failure is provided at the cellular level by NK cells, which our genetic analysis had indicated were sufficient to mediate pregnancy loss in the absence of other lymphoid lineages, and at the molecular level by TNF- $\alpha$, which was required for pregnancy loss likely through its direct effects on ovarian function. Indeed, TNF- $\alpha$ is produced upon the cellular activation of both NK cells and dendritic cells and is thought to play an important role as an amplifier of the reciprocal interactions between the two cell types (32). Consistent with our model, TNF- $\alpha$ has previously been shown to inhibit luteal steroidogenesis in vitro $(13,33)$.

Although our results strongly suggest this straightforward epistatic relationship between the immune and endocrine systems, it remains possible that more complex interactions are taking place in a manner consistent with a potential immunosuppressive role for progesterone during pregnancy $(16,34)$. However, we consider this to be very unlikely, because we found that concurrent treatment of mice with pharmacological doses of progesterone did not alter the systemic immune activation or the ovarian upregulation of IL-6, IKB $\alpha$, Socs 1 , and Socs3 following CD40 ligation, even though it prevented pregnancy failure. Furthermore, exogenous prolactin also rescued FGK45-induced pregnancy failure, yet only raised serum progesterone concentrations to subphysiological levels and had no effect on FGK45-induced lymphoid activation (data not shown). Lastly, the progesterone antagonist RU486 is

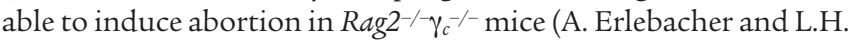
Glimcher, unpublished observations), which demonstrates that the lymphoid system is not required for pregnancy failure following endocrine withdrawal.

The pathway described here also stands in contrast to the local processes at the maternal-fetal interface that are currently thought to underlie other examples of immune-mediated pregnancy loss in rodents (1). For example, systemically injected anti-phospholipid antibodies, LPS, or inflammatory cytokines such as TNF- $\alpha$ or IFN- $\gamma$ are thought to induce abortion or modulate embryo resorption rates through localized actions within implantation sites with direct damage to the fetus and placenta or deranged placental and decidual hemostasis emerging as possible common pathogenic mechanisms (1,9-11). Although many of these agents would be expected to invoke inflammatory cytokine cascades similar to those produced by CD40 ligation, some have been shown to be effective throughout gestation, with their actions ultimately resistant to progesterone injection $(10,35)$. Thus, it is possible that some agents

\section{Figure 5}

Involvement of TNF- $\alpha$ in anti-CD40-induced pregnancy failure. (A) Elevated TNF- $\alpha$ and IL-6 serum concentrations 24 hours after treatment with anti-CD40 antibodies (FGK45). Data represent mean \pm SEM of five mice per group. The limits of detection were $31.1 \mathrm{pg} / \mathrm{ml}$ TNF- $\alpha$ and 54.7 $\mathrm{pg} / \mathrm{ml}$ IL-6. ND, not detectable. (B and C) NF-kB p65 distribution in E4 corpora lutea visualized by immunostaining. Whereas the $\mathrm{p} 65$ distribution was cytoplasmic in mice treated 24 hours earlier on E4 with control rat $\lg G(B)$, it assumed a nuclear distribution when mice were treated with FGK45 (C). Data represent immunostained ovaries from three mice per group. Scale bar: $50 \mu \mathrm{m}$.
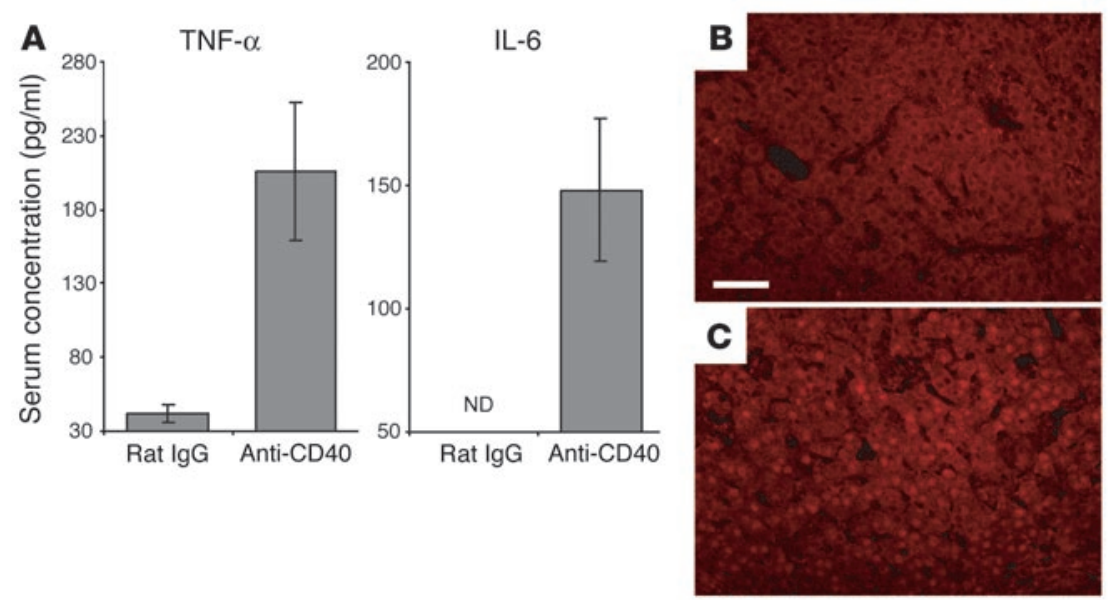


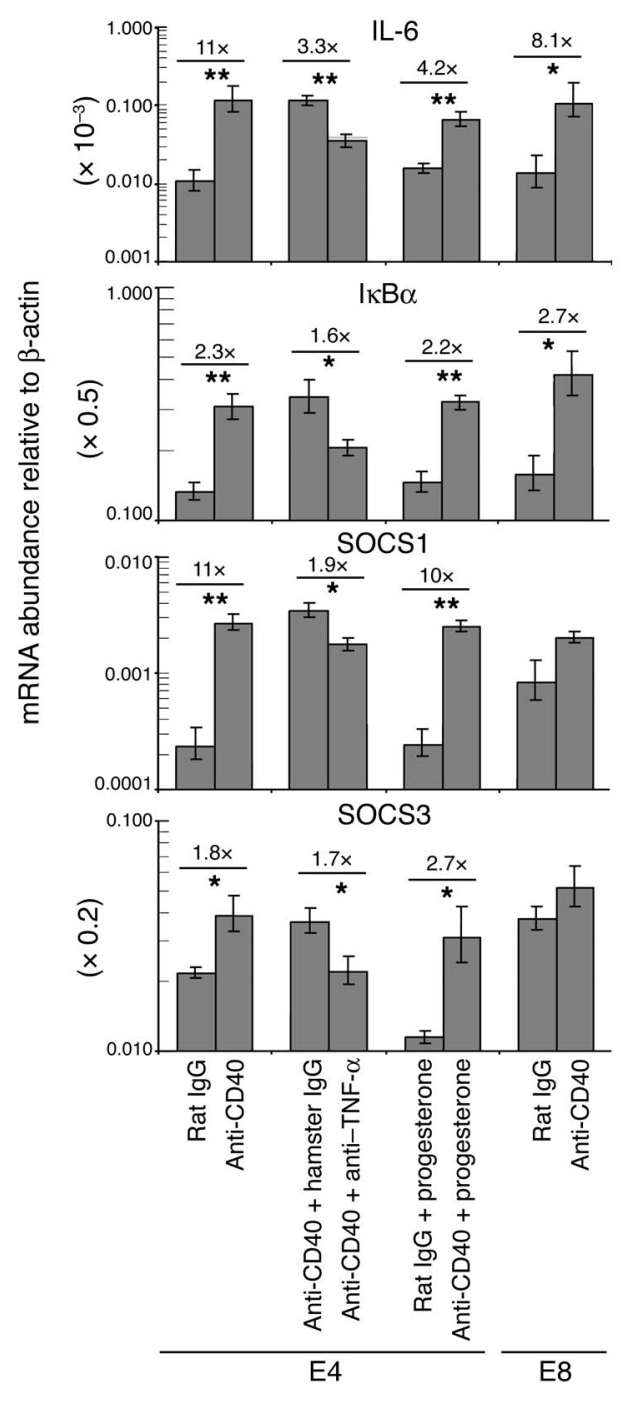

cause pregnancy failure by two independent but superimposed mechanisms: systemic inhibition of ovarian function, and local induction of inflammatory processes within implantation sites. In support of this idea, we have found that concurrent progesterone administration grossly prevented the complete fetal resorption and decidual sloughing caused by LPS injection early in gestation, although implantation sites showed large areas of hemorrhage (A. Erlebacher and L.H. Glimcher, unpublished observations).

Molecular mechanisms of luteal insufficiency. Ovarian mRNA expression studies suggested that the luteal insufficiency following CD40 ligation was likely due to a number of quantitative changes in gene expression, including upregulated $20 \alpha$-HSD expression on E5 and particularly E8, leading to increased intraovarian progesterone catabolism, and downregulated $3 \beta$-HSD expression on E5, leading to decreased progesterone synthesis. Furthermore, downregulated LHR expression on both E5 and E8 might lead to decreased posttranslational activation of $\operatorname{StAR}(33,36)$, and decreased prolactin or LH signaling in either corpus luteal cells or ovarian endothelial cells might cause additional developmental or metabolic defects that secondarily impair progesterone production (14).

While decreased LHR mRNA expression might be due in part to a direct effect of TNF- $\alpha$ as described previously (37), the constella-

\section{Figure 6}

Ovarian mRNA expression of IL-6, IкB $\alpha$, Socs1, and Socs3 upon systemic CD40 ligation. Whole-ovary mRNA expression levels were determined by real-time RT-PCR. Ovaries were collected from mice on E4 12 hours after treatment with rat IgG or FGK45 ( $n=4$ mice per group); with FGK45 plus control hamster IgG or FGK45 plus TNF- $\alpha$-neutralizing mAb's ( $n=5$ mice per group); or with rat IgG plus progesterone or FGK45 plus progesterone ( $n=4$ mice per group). Ovaries were collected on E8 12 hours after injection of rat IgG or FGK45 ( $n=3$ mice per group). Data represent mean \pm SEM. On E4, IL-6, IKB $\alpha$, Socs1, and Socs3 mRNA levels were increased following FGK45 injection $\left({ }^{\star} P<0.05 ;{ }^{* \star} P<0.005\right)$. These increases were inhibited by TNF- $\alpha$ blockade but not prevented by concurrent progesterone treatment. On E8, FGK45 injection led to upregulated mRNA expression of IL-6 and $I_{\kappa} B \alpha$, but not Socs1 or Socs3. For ease of graphical representation, transcript levels for IL-6, I $\mathrm{KB} \alpha$, and Socs3 were multiplied by $1,000,2$, and 5 , respectively.

tion of LHR downregulation and $20 \alpha-H S D$ upregulation suggested a more general defect in the ovarian prolactin response. Since serum prolactin levels were unimpaired and ovarian prolactin receptor and STAT5 mRNA levels were largely maintained, the defect likely involved dominant inhibition of postreceptor signal transduction. We suggest that this inhibitory function can be ascribed at least in part to the cytokine-inducible JAK/STAT signaling inhibitors Socs1 and Socs3. In prior studies, both Socs 1 and Socs 3 have been shown to inhibit prolactin receptor signaling via STAT5 in vitro (38-40), and a genetic interaction between Socs 1 and prolactin receptor signaling in vivo has been shown by the ability of Socs 1 heterozygosity to rescue the lactation defect seen in $\mathrm{Prlr}^{+/-}$mice (41). This result also emphasizes how final STAT5 signal strength can be profoundly influenced by as little as presumably twofold changes in the relative levels of Socs proteins and signaling components. Lastly, luteal induction of Socs1 and in particular Socs 3 following prostaglandin F- $2 \alpha$ administration in rats has recently been postulated to explain the luteolytic effects of this prostaglandin and its ability to inhibit prolactin signaling (42). In our system, both Socs1 and Socs3 were acutely upregulated in the ovaries following systemic CD40 ligation, and this upregulation could be partially (Socs1) or largely (Socs3) inhibited by concomitant TNF- $\alpha$ blockade, a treatment that ultimately prevented pregnancy failure. The inducibility of these factors following CD40 ligation also temporally correlated with the postimplantation window of sensitivity toward FGK45-induced pregnancy failure.

It should be emphasized, however, that the cellular and molecular mechanisms of immune-mediated luteal insufficiency are likely to be overlapping and manifold. TNF- $\alpha$ is a product of immune cell populations besides NK cells and dendritic cells, and the actions of TNF- $\alpha$ might be indirectly mediated, for example through the induction of prostaglandin F-2 $\alpha$, a luteolytic agent also induced by other inflammatory cytokines $(13,33)$. Furthermore, inflammatory cytokines besides TNF- $\alpha$ are able to inhibit luteal steroidogenesis in vivo and in vitro (e.g., IL-1 and IFN- $\gamma)(13,33,43)$, and many cytokines are able to induce Socs protein expression $(22,23)$. Thus, it is likely that multiple inflammatory pathways induced by a variety of stimuli might lead to luteal insufficiency and endocrine failure in the absence of TNF- $\alpha$, or contribute to luteal insufficiency in the presence of TNF- $\alpha$. Indeed, the only partial ability of TNF- $\alpha$ blockade to reverse Socs1 upregulation points to a role for other cytokines in luteal insufficiency even in our system. Further experiments will be necessary to define the precise roles of Socs proteins in the immune regulation of ovarian function. 
Since prolactin is not thought to be a major stimulus for progesterone production in humans, it is not immediately clear how mechanisms of suppressed prolactin receptor signaling would be directly relevant to disorders of human reproduction. Progesterone production by the corpus luteum early in human pregnancy (up to about week 8) is driven by human chorionic gonadotropin (hCG) produced by the placenta, while later in gestation the placenta itself becomes the primary source of progesterone, likely stimulated by hCG acting in an autocrine fashion (16). Furthermore, hCG and LH bind to a shared G protein-coupled receptor, and so these hormones have been thought to signal largely through cAMP second messengers. However, there has been increasing evidence that signaling via $G$ protein-coupled receptors also induces JAK/STAT activation (42), and a recent report has shown that the rat LHR couples to JAK2/STAT1 and JAK2/STAT5 in the ovaries (44). Thus, we might expect that hCG/LH signaling in the corpus luteum or placenta would be sensitive to SOCS inhibition, and this might explain the in vitro inhibitory effects of TNF- $\alpha$ and other inflammatory cytokines on LH- and hCG-stimulated luteal steroidogenesis that have been previously noted in many species (13). Intriguingly, ovarian resistance towards endogenous LH or exogenous hCG has been associated clinically with luteal insufficiency in humans (45).

Thus, it may be worth considering the possibility that immune activation contributes to human infertility and in particular occult or recurrent pregnancy loss through the negative effects on the reproductive endocrine system that we describe here. For example, activation of the CD40 pathway might occur through CD40L upregulation by either activated T cells or activated NK cells in response to infection or to potentially transformed or overinvasive trophoblasts at the maternal-fetal interface. Interestingly, complement $\mathrm{C} 4 \mathrm{~b}$-binding protein $(\mathrm{C} 4 \mathrm{BP})$, produced in part by activated monocytes, has recently been shown to be another activating ligand for CD40 (46). This protein, which also regulates the complement and coagulation cascades, might provide a nonlymphoid stimulus for CD40 activation at sites of inflammation. Although we do not know whether NK cell activation or TNF- $\alpha$ production alone is sufficient to mediate the full effect of CD40 ligation, we would expect these intermediates to cause some level of endocrine dysfunction as well.

\section{Methods}

Mice, antibody treatments, and hormone treatments. CD40-/- mice were the gift of R. Geha (Harvard Medical School). C57BL/6 (B6), BALB/c, B6CBAF1, and B6129F1 mice (the latter being a strain-matched control for $\mathrm{Cd} 40^{-/-}$mice) were from The Jackson

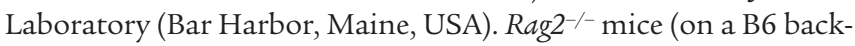
ground) and $R a g 2^{-1-} \gamma_{c}^{-1-}$ mice (on a mixed C57BL/6 $\times$ C57BL/10 background) were from Taconic (Germantown, New York, USA). Males were mated with individual females no more than once per week; B6CBAF1 females and B6 males were used in all experiments except when noted. The day of the copulation plug was counted as E0. All antibodies were given via intraperitoneal injection at the dose of $100 \mu \mathrm{g}$ in $0.1 \mathrm{ml}$ PBS. The FGK45 rat hybridoma cell line was the gift of A. Groenewegen (Basel Institute for Immunology, Basel, Switzerland); antibodies were prepared from hybridoma supernatants by protein $\mathrm{G}$ chromatography either in house or by BioExpress Cell Culture Services (West Lebanon, New Hampshire, USA). Polyclonal rat IgG antibodies were purchased from ICN Biomedicals Inc. (Aurora, Ohio, USA). Mice received FGK45 or rat
IgG antibodies between 0830 and 1200 hours. For experiments evaluating the role of TNF- $\alpha$, mice were additionally injected 2 hours later with TN3-19.12, a hamster IgG mAb that neutralizes murine TNF- $\alpha$ (47), or $100 \mu \mathrm{g}$ PIP, an irrelevant hamster IgG mAb that recognizes glutathione-S-transferase. Both of these antibodies were the gifts of R. Schreiber (Washington University, St. Louis, Missouri, USA). Progesterone (Sigma-Aldrich, St. Louis, Missouri, USA) was dissolved in sesame seed oil (Sigma-Aldrich), and 2 $\mathrm{mg} / 0.1 \mathrm{ml}$ was injected subcutaneously at the time of concurrent antibody injections. Ovine prolactin (NIDDK-oPRL-21, produced by the National Hormone and Peptide Program, Torrance, California, USA) was dissolved in alkaline saline, and $100 \mu \mathrm{g} / 0.1 \mathrm{ml}$ was injected subcutaneously twice a day between $0800-0830$ hours and 1600-1630 hours. All mouse experiments were approved by Harvard Medical School's Standing Committee on Animals.

Serum hormone and cytokine measurements. Serum was collected by retro-orbital eye bleeds. Progesterone concentrations were measured by enzyme-linked immunoassay (ALPCO Diagnostics, Windham, New Hampshire, USA), prolactin and LH concentrations were measured by RIA in the laboratory of the National Hormone and Peptide Program, and cytokines were measured by the SearchLight assay (Pierce Biotechnology Inc., Rockford, Illinois, USA).

Flow cytometry. Splenocyte forward scatter and cell surface marker expression was determined by flow cytometry using a BD Biosciences FACSCalibur and CellQuest software (San Diego, California, USA). The following antibodies were purchased from BD Biosciences: CyChrome-conjugated anti-TCR- $\beta$ (clone H57-597), FITC-anti-NK1.1 (PK136), phycoerythrin-anti-B220 (RA3-6B2), phycoerythrin-anti-CD69 (H1.2F3), allophycocyanin-anti-CD11c (HL3), phycoerythrin-anti-CD11b (M1/70), FITC-anti-CD80 (1610A1), FITC-anti-CD86 (GL1), FITC-anti-MHC class II (AF6120.1), and FITC-anti-CD40 (HM40-3). Following an initial gating on all lymphoid cells based on their forward and side scatter characteristics, T cells were identified as the TCR- $\beta^{+} \mathrm{NK} 1.1^{-}$population, NK cells were identified as the TCR- $\beta{ }^{-} \mathrm{NK} 1.1^{+}$population, and $\mathrm{B}$ cells were identified as the TCR- $\beta{ }^{-} \mathrm{NK} 1.1^{-}$population. True B $220^{+}$B cells comprised $70-80 \%$ of this latter population in rat IgG-treated mice, and 85-95\% of this latter population in FGK45treated mice. Dendritic cells were identified and gated by virtue of their expression of CD11c, with an unused fluorescence channel employed to gate away autofluorescent cells.

Histology and immunofluorescence microscopy. Tissue was fixed overnight at $4{ }^{\circ} \mathrm{C}$ in $4 \%$ paraformaldehyde prior to routine paraffin embedding. For leukocyte visualization, sections were stained with biotin-conjugated antibodies against the common leukocyte antigen CD45 (clone 30-F11; BD Biosciences), using HRP-conjugated streptavidin as a secondary reagent (NEN Life Science Products Inc., Boston, Massachusetts, USA), biotin-tyramide amplification (NEN Life Science Products Inc.), and streptavidin-Alexa 594 (Molecular Probes Inc., Eugene, Oregon, USA) as the final fluorochrome. Sections were counterstained with DAPI (SigmaAldrich) and digitally photographed at $\times 4$ magnification. Composites were assembled in Adobe Photoshop (Adobe Systems Inc., San Jose, California, USA). NF-кB p65 was visualized with a goat primary antibody (C-20; Santa Cruz Biotechnology Inc., Santa Cruz, California, USA), a biotin-conjugated horse anti-goat secondary antibody (Vector Laboratories Inc., Burlingame, California, USA), and streptavidin-Alexa 594.

RNA analysis. RNA was prepared from homogenized ovaries using the Trizol reagent (Invitrogen Corp., Carlsbad, California, 
USA). Real-time RT-PCR was performed as described previously (48). All reactions were run in duplicate using cDNA template synthesized from $10 \mathrm{ng}$ RNA. Difference in threshold cycle $\left(\Delta \mathrm{C}_{\mathrm{T}}\right)$ values were calculated relative to $\beta$-actin, and statistical analyses were performed on groups of raw $\Delta C_{T}$ values. Expression levels relative to $\beta$-actin were calculated as $2^{-\Delta C_{\mathrm{T}}}$, with error bars extrapolated from the SD of the $\Delta \mathrm{C}_{\mathrm{T}}$ mean for each group. We used the following primer/probe sets, listed $5^{\prime}$ to $3^{\prime}$ in the order of forward primer, reverse primer, and (when used) probe: $\beta$-actin, GCTCTGGCTCCTAGCACCAT, GCCACCGATCCACACCGCGT, TCAAGATCATTGCTCCTCCTGAGCGC; StAR, TCACTTGGCTGCTCAGTATTGAC, TCTATCTGGGTCTGCGATAGGAC; P450scc, CGGTACTTGGGCTTTGGCT, AGCAGATTGATAAGGAGGATGGTC; $3 \beta$-HSD, CCAGGCAGACCATCCTAGATGT, AGATGAAGGCTGGCACACTTG; LHR, CGAGACGCTTTATTCTGCCAT, AGCATCTGGTTCTGGAGTACATTG; $20 \alpha$-HSD, GCTGATATGTTTAAGGCTCACCCTAA, AGAGTCCAGCATCACACAAAAGATC; prolactin receptor, GGATGTGACTTACATTGTTGAACCA, TACCCACAGATATGTTTTTTTGTCTTTT; STAT5a, TGGTCCCTGAGTTCGTCAATG, GTTGAGGGCACACGACTGG; STAT5b, GCGCCACCTACATGGATCA, GACGGAGTCCGGGTTGG; IL-6, TTCAACCAAGAGGTAAAAGATTTACATAA, CACTCCTTCTGTGACTCCAGCTT; I B $\alpha$, AGATGCTACCCGAGAGCGAG, TCATCATAGGGCAGCTCATCC; Socs1, TGTGCCGCAGCATTAAGTG, GGCATCTCACCCTCCACAAC, CCGCCTGGGTCGGAGGGAGT; Socs3, TGCTGGCCAAAGAAATAACCA, GGT-
CACCCCTTGCCACTCT, CCCACTGCCCAGCCTAGGTGAGGA. All probes were dual-labeled with FAM and TAMRA. SYBR green was used for amplifications when a primer set was used without a probe. For all primer/probe sets, the approximate difference in amplification between RT and mock RT reactions was greater than 1,000-fold, except the SYBR green amplifications of $\beta$-actin (50-fold), STAT5a (100-fold), and STAT5b (50-fold).

Statistical analysis. Pregnancy rates were compared by Fisher's exact test; all other comparisons used Student's $t$ test.

\section{Acknowledgments}

We are grateful to A. Groenewegen, R. Schreiber, and R. Geha for valuable reagents and thank K. Price, L. Kangaloo, and C. McCall for technical and administrative assistance, J. Strauss, J. Boyson, M. Townsend, and members of the Glimcher laboratory for valuable discussions, and G. Lord and D. Schust for critical reading of the manuscript. This work was supported by NIH grants AI01650 and AI54370.

Received for publication November 25, 2003, and accepted in revised form April 20, 2004.

Address correspondence to: Adrian Erlebacher, Department of Immunology and Infectious Diseases, Harvard School of Public Health FXB-205, 651 Huntington Avenue, Boston, Massachusetts 02115, USA. Phone: (617) 432-0924; Fax: (617) 432-0084; E-mail: aerlebac@hsph.harvard.edu.
1. Laird, S.M., et al. 2003. A review of immune cells and molecules in women with recurrent miscarriage. Hum. Reprod. Update. 9:163-174.

2. Aoki, K., et al. 1995. Preconceptional natural-killercell activity as a predictor of miscarriage. Lancet. 345:1340-1342.

3. Coulam, C.B., Goodman, C., Roussev, R.G., Thomason, E.J., and Beaman, K.D. 1995. Systemic CD56+ cells can predict pregnancy outcome. Am. J. Reprod. Immunol. 33:40-46.

4. Kwak, J.Y., et al. 1995. Up-regulated expression of $\mathrm{CD} 56^{+}, \mathrm{CD} 6^{+} / \mathrm{CD} 16^{+}$, and $\mathrm{CD} 19^{+}$cells in peripheral blood lymphocytes in pregnant women with recurrent pregnancy losses. Am. J. Reprod. Immunol. 34:93-99.

5. Emmer, P.M., et al. 2000. Peripheral natural killer cytotoxicity and $\mathrm{CD} 56^{+} \mathrm{CD} 16^{+}$cells increase during early pregnancy in women with a history of recurrent spontaneous abortion. Hum. Reprod. 15:1163-1169.

6. Ntrivalas, E.I., et al. 2001. Status of peripheral blood natural killer cells in women with recurrent spontaneous abortions and infertility of unknown aetiology. Hum. Reprod. 16:855-861.

7. Raghupathy, R. 1997. Th1-type immunity is incompatible with successful pregnancy. Immunol. Today. 18:478-482.

8. Piccinni, M.P., et al. 1998. Defective production of both leukemia inhibitory factor and type 2 $\mathrm{T}$-helper cytokines by decidual $\mathrm{T}$ cells in unexplained recurrent abortions. Nat. Med. 4:1020-1024.

9. Gendron, R.L., Nestel, F.P., Lapp, W.S., and Baines, M.G. 1990. Lipopolysaccharide-induced fetal resorption in mice is associated with the intrauterine production of tumour necrosis factor-alpha. J. Reprod. Fertil. 90:395-402.

10. Clark, D.A., Chaouat, G., Arck, P.C., Mittruecker, H.W., and Levy, G.A. 1998. Cytokine-dependent abortion in CBA $x$ DBA/ 2 mice is mediated by the procoagulant fgl2 prothrombinase. J. Immunol. 160:545-549.

11. Holers, V.M., et al. 2002. Complement C3 activa- tion is required for antiphospholipid antibodyinduced fetal loss. J. Exp. Med. 195:211-220.

12. Rivest, S., and Rivier, C. 1995 . The role of corticotropin-releasing factor and interleukin- 1 in the regulation of neurons controlling reproductive functions. Endocr. Rev. 16:177-199.

13. Davis, J.S., and Rueda, B.R. 2002. The corpus luteum: an ovarian structure with maternal instincts and suicidal tendencies. Front. Biosci. 7:d1949-d1978.

14. Risk, M., and Gibori, G. 2001. Mechanisms of luteal cell regulation by prolactin. In Prolactin. N.D. Horseman, editor. Kluwer Academic Publishers. Boston, Massachusetts, USA. 265-295.

15. Deanesly, R. 1973. Termination of early pregnancy in rats after ovariectomy is due to immediate collapse of the progesterone-dependent decidua. J. Reprod. Fertil. 35:183-186.

16. Jaffe, R.B. 1999. Neuroendocrine-metabolic regulation of pregnancy. In Reproductive endocrinology. S.S.C. Yen, R.B. Jaffe, and R.L. Barbieri, editors. W.B. Saunders Co. Philadelphia, Pennsylvania, USA. 751-784.

17. Raj, H.G., and Moudgal, N.R. 1970. Hormonal control of gestation in the intact rat. Endocrinology. 86:874-889.

18. Mednick, D.L., Barkley, M.S., and Geschwind, I.I. 1980. Regulation of progesterone secretion by LH and prolactin during the first half of pregnancy in the mouse. J. Reprod. Fertil. 60:201-207.

19. Diehl, L., et al. 2000. The role of CD40 in peripheral T cell tolerance and immunity. J. Mol. Med. 78:363-371.

20. van Kooten, C., and Banchereau, J. 2000. CD40CD40 ligand. J. Leukoc. Biol. 67:2-17.

21. Auernhammer, C.J., and Melmed, S. 2001. The central role of SOCS-3 in integrating the neuroimmunoendocrine interface. J. Clin. Invest. 108:1735-1740. doi:10.1172/JCI200114662.

22. Alexander, W.S. 2002. Suppressors of cytokine signalling (SOCS) in the immune system. Nat. Rev. Immunol. 2:410-416.
23. Shuai, K., and Liu, B. 2003. Regulation of JAKSTAT signalling in the immune system. Nat. Rev. Immunol. 3:900-911.

24. Rolink, A., Melchers, F., and Andersson, J. 1996. The SCID but not the RAG-2 gene product is required for $\mathrm{S}$ mu-S epsilon heavy chain class switching. Immunity. 5:319-330.

25. Castigli, E., et al. 1994. CD40-deficient mice generated by recombination-activating gene-2-deficient blastocyst complementation. Proc. Natl. Acad. Sci. U. S. A. 91:12135-12139.

26. Rubinstein, L., and Forbes, T.R. 1963. Maintenance of pregnancy with subcutaneous pellets of progesterone in ovariectomized mice. Proc. Soc. Exp. Biol. Med. 113:1043-1046.

27. Stocco, C., Callegari, E., and Gibori, G. 2001. Opposite effect of prolactin and prostaglandin F- $2 \alpha$ on the expression of luteal genes as revealed by rat cDNA expression array. Endocrinology. 142:4158-4161.

28. Markoff, E., and Talamantes, F. 1981. Serum placental lactogen in mice in relation to day of gestation and number of conceptuses. Biol. Reprod. 24:846-851.

29. Shinkai, Y., et al. 1992. RAG-2-deficient mice lack mature lymphocytes owing to inability to initiate V(D)J rearrangement. Cell. 68:855-867.

30. Cao, X., et al. 1995. Defective lymphoid development in mice lacking expression of the common cytokine receptor gamma chain. Immunity. 2:223-238.

31. Ghosh, S., May, M.J., and Kopp, E.B. 1998. NFkappa B and Rel proteins: evolutionarily conserved mediators of immune responses. Annu. Rev. Immunol. 16:225-260.

32. Moretta, A. 2002. Natural killer cells and dendritic cells: rendezvous in abused tissues. Nat. Rev. Immunol. 2:957-964.

33. Niswender, G.D., Juengel, J.L., Silva, P.J., Rollyson, M.K., and McIntush, E.W. 2000. Mechanisms controlling the function and life span of the corpus luteum. Physiol. Rev. 80:1-29. 
34. Stites, D.P., and Siiteri, P.K. 1983. Steroids as immunosuppressants in pregnancy. Immunol. Rev. 75:117-138.

35. Gasic, G.J., Gasic, T.B., and Strauss, J.F. 1975. Abortifacient effects of Vibrio cholerae exo-enterotoxin and endotoxin in mice. J. Reprod. Fertil. 45:315-322.

36. Arakane, F., et al. 1997. Phosphorylation of steroidogenic acute regulatory protein (StAR) modulates its steroidogenic activity. J. Biol. Chem. 272:32656-32662.

37. Chen, Y.J., Feng, Q., and Liu, Y.X. 1999. Expression of the steroidogenic acute regulatory protein and luteinizing hormone receptor and their regulation by tumor necrosis factor alpha in rat corpora lutea. Biol. Reprod. 60:419-427.

38. Pezet, A., Favre, H., Kelly, P.A., and Edery, M. 1999. Inhibition and restoration of prolactin signal transduction by suppressors of cytokine signaling. J. Biol. Chem. 274:24497-24502.

39. Dif, F., Saunier, E., Demeneix, B., Kelly, P.A., and Edery, M. 2001. Cytokine-inducible SH2-contain- ing protein suppresses PRL signaling by binding the PRL receptor. Endocrinology. 142:5286-5293.

40. Tam, S.P., Lau, P., Djiane, J., Hilton, D.J., and Waters, M.J. 2001. Tissue-specific induction of SOCS gene expression by PRL. Endocrinology. 142:5015-5026.

41. Lindeman, G.J., et al. 2001. SOCS1 deficiency results in accelerated mammary gland development and rescues lactation in prolactin receptordeficient mice. Genes Dev. 15:1631-1636.

42. Curlewis, J.D., et al. 2002. A prostaglandin F-2 $\alpha$ analog induces suppressors of cytokine signaling3 expression in the corpus luteum of the pregnant rat: a potential new mechanism in luteolysis. Endocrinology. 143:3984-3993.

43. Liu, Y.X., Chen, Y.X., Shi, F.W., and Feng, Q. 1995. Studies on the role of plasminogen activators and plasminogen activator inhibitor type- 1 in rat corpus luteum of pregnancy. Biol. Reprod. 53:1131-1138.

44. Carvalho, C.R., et al. 2003. Novel signal transduction pathway for luteinizing hormone and its interaction with insulin: activation of Janus kinase/signal transducer and activator of transcription and phosphoinositol 3-kinase/Akt pathways. Endocrinology. 144:638-647.

45. Hinney, B., Henze, C., Kuhn, W., and Wuttke, W. 1996. The corpus luteum insufficiency: a multifactorial disease. J. Clin. Endocrinol. Metab. 81:565-570.

46. Brodeur, S.R., et al. 2003. C4b-binding protein (C4BP) activates B cells through the CD40 receptor. Immunity. 18:837-848.

47. Sheehan, K.C., Ruddle, N.H., and Schreiber, R.D. 1989. Generation and characterization of hamster monoclonal antibodies that neutralize murine tumor necrosis factors. J. Immunol. 142:3884-3893.

48. Erlebacher, A., Lukens, A.K., and Glimcher, L.H. 2002. Intrinsic susceptibility of mouse trophoblasts to natural killer cell-mediated attack in vivo. Proc. Natl. Acad. Sci. U. S. A. 99:16940-16945. 